\title{
On Some Aspects of an Hg Based EPR Experiment*
}

\author{
Thomas Walther and Edward S. Fry \\ Physics Department, Texas A \& M University, College Station, TX 77843-4242, USA
}

Z. Naturforsch. 52a, 20-24 (1997); received October 12, 1995

\begin{abstract}
An experimental realization of Bohm's spin-1/2 EPR gedanken-experiment is possible using the spin-1/2 nuclei of the isotope ${ }^{199} \mathrm{Hg}$. The dissociation of dimers of the ${ }^{199} \mathrm{Hg}_{2}$ isotopomer, using a spectroscopically selective stimulated Raman process, leads to the generation of an entangled state between the two ${ }^{199} \mathrm{Hg}$ atoms. The measurement of nuclear spin correlations between the two atoms in this entangled state is achieved by detection of the atoms using a spin state selective two photon excitation-ionization scheme. The experiment will not only close the detector efficiency loophole, but in addition will permit enforcement of the locality condition. Effects of imperfect analyzers will also be discussed.
\end{abstract}

\section{Introduction}

In 1951 Bohm introduced a version of the EPR gedanken-experiment, which was based on two separated spin- $1 / 2$ particles in an entangled singlet state. The spin states of the two particles were to be observed with two sets of Stern-Gerlach magnets tilted with respect to each other. For this system John Bell proved that any hidden variable theory satisfying a physically reasonable condition of locality will yield predictions that restrict the statistical correlations between the spin-states of the two spin-1/2 particles [1]. Mathematically this behavior can be expressed in the form of inequalities. Furthermore Bell demonstrated that quantum mechanics yields statistical predictions that can violate these restrictions. Thus for the first time experimental tests concerning the EPR-paradox [2] were conceivable. However, any experimental imperfection, such as detector efficiencies less than unity, leads to quantum mechanical predictions that do not violate the inequalities. Thus, additional auxiliary assumptions had to be introduced in order to make physically realizable experiments possible with existing technology [3].

An inequality that requires no auxiliary assumptions and is therefore called a strong Bell (BCH) inequality was obtained by Clauser and Horne in 1974 $[4,5]$. They derived this inequality in the context of Bohm's gedanken-experiment with the Stern-Gerlach

\footnotetext{
* Presented at a Workshop in honor of E. C. G. Sudarshan's contributions to Theoretical Physics, held at the University of Texas in Austin, September 15-17, 1991.

Reprint requests to Dr. T. Walther.
}

magnets replaced by simple analyzers (c.f. Figure 1). The resulting inequality is formulated in terms of ratios of experimentally accessible coincidence and singles count rates for the two particles in various spin states. But although an experiment is feasible, there are still restrictions on the experimental setup. High efficiency detectors and high discrimination of the analyzer system are requirements for a test of the quantum mechanical predictions against this inequality. The $\mathrm{BCH}$ inequality has not yet been tested experimentally.

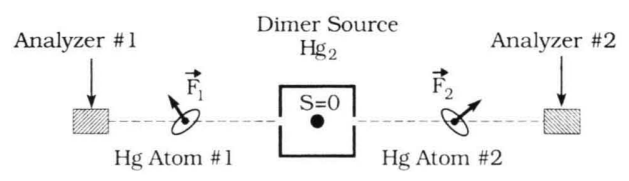

Fig. 1. Schematic setup of Bohm's version of the EPR gedanken-experiment using the singlet state of two spin-1/2 particles. The Hg-isotope ${ }^{199} \mathrm{Hg}$ allows a realization of this gedanken-experiment.

Recently we described a detailed proposal for such an experiment [6]. We restrict the discussion here to two important aspects; one is the generation of the entangled state between the spin- $1 / 2$ particles, and the second is the extension of the discussion to non-perfect analyzers.

\section{Entangled State Generation}

We consider a homogenous molecule, in which each atom has nuclear spin $1 / 2$ and for which the total 
molecular electron and nuclear spin angular momenta are zero. The wave function for such a molecule can be expressed in terms of the separated atom basis states as [6]

$$
|\Psi\rangle=\frac{1}{\sqrt{2}}\left\{(\uparrow)_{1}(\downarrow)_{2}-(\downarrow)_{1}(\uparrow)_{2}\right\}
$$

This is identical to the state of the two spin $1 / 2$ particles in Bohm's version of the EPR gedanken-experiment [4]. Thus, a spatially separated two atom system, for example prepared by photo-dissociation of this molecule, is in an "entangled" state suitable for testing Bell inequalities.

In our experiment spatial separation is achieved by dissociation of ${ }^{199} \mathrm{Hg}_{2}$ dimers in a supersonic beam via stimulated Raman excitation to a dissociating state of their $X^{1} \Sigma_{\mathrm{g}}^{+}$ground state (c.f. Figure 2). First a laser system at $266 \mathrm{~nm}$ drives a transition in the $v$ 58-0 band of the $D^{3} \Sigma_{\mathrm{u}}^{+} \leftarrow X^{1} \Sigma_{\mathrm{g}}^{+}$system. A second laser at $355 \mathrm{~nm}$ completes a stimulated Raman transition to a continuum level of the ground state and leaves the dissociated atoms with a center-of-mass (CM) kinetic energy of $1.17 \mathrm{eV}$. Since the experiment requires that two ${ }^{199} \mathrm{Hg}$ atoms are in an entangled state with total nuclear spin $I=0$, only the dimers with a total nuclear spin $I=0$ must be dissociated.

Since ${ }^{199} \mathrm{Hg}$ is a fermion with nuclear spin $1 / 2$, the effects of nuclear spin statistics on the selection rules must be taken into account in the excitation/dissociation process. The question arises, do the selection rules for the aforementioned transition in a ${ }^{199} \mathrm{Hg}_{2}$ dimer make it possible to selectively excite only those dimers which are in a nuclear spin singlet state?

Since they are fermions, the ${ }^{199} \mathrm{Hg}_{2}$ dimers must have an anti-symmetric total wave function with re-

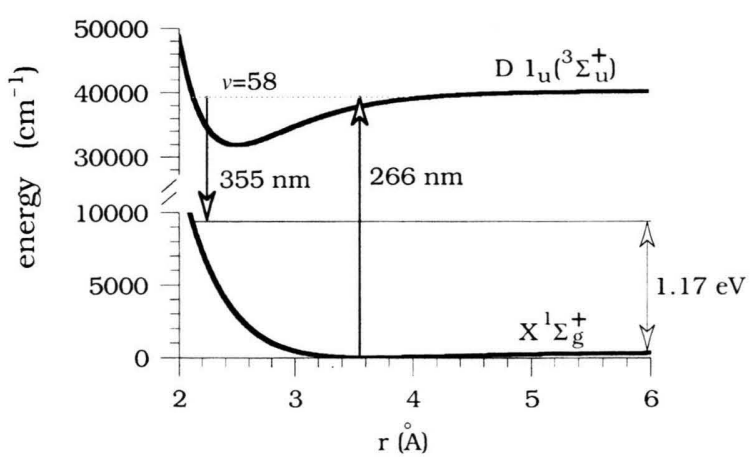

Fig. 2. Energy levels of a mercury dimer and the relevant Raman transitions leading to dissociation. Note the shift in scale on the ordinate. spect to an exchange of the two nuclei in order to conform to the Pauli principle. An exchange of the two nuclei is equivalent to an inversion of the total wave function at the origin, followed by a rotation of the electronic wave function by $180^{\circ}$ and a reflection of the electronic wave function in a plane containing the two nuclei [7]. It should be noted that the electronic wave function for a diatomic molecule is not altered by a $180^{\circ}$ rotation since it only depends on the relative coordinates of the electrons with respect to the nuclei.

The overall symmetry of the wave function is given by the combination of the symmetries of the nuclear spin, vibrational, rotational, and electronic wave functions, respectively. First, the two spin $1 / 2$ nuclei of ${ }^{199} \mathrm{Hg}_{2}$ form either a nuclear spin singlet (anti-symmetric, total nuclear spin $I=0$ ), or spin triplet (symmetric, $I=1$ ). Second, the vibrational part is always symmetric, since it only depends on the relative nuclear coordinates. Third, the symmetry of the rotational wave function is $(-1)^{N}$, where $N$ is the rotational quantum number; it is symmetric for even, and anti-symmetric for odd rotational quantum numbers. Fourth, the electronic wave function has the symmetry of the electronic state.

The electronic ground state of ${ }^{199} \mathrm{Hg}_{2}$ is a ${ }^{1} \Sigma_{\mathrm{g}}^{+}$ state; $g$ signifies it is symmetric with respect to an inversion of the electronic wave function at the origin and + signifies it is symmetric with respect to a reflection in a plane containing the two nuclei. Thus, since the electronic and vibrational wave functions are symmetric, the combination of rotational and nuclear spin states must be anti-symmetric in order to have an overall anti-symmetric wave function. Specifically, in the ground electronic state, nuclear spin singlet states are associated with even $N$, and triplet states with odd $N$, rotational levels [7].

In contrast, the electronic excited state ${ }^{3} \Sigma_{u}^{+}\left(1_{u}\right)$ is anti-symmetric with respect to an inversion of the electronic wave function at the origin. Hence the combination of rotational and nuclear spin states must be symmetric. Specifically, in this state, the nuclear spin singlet states are associated with the odd $N$ levels, and the nuclear triplet states with the even $N$ levels [7]. The fact that the electronic state is a triplet state with respect to the electron spin does not alter these symmetry considerations.

Now the selection rule for these molecular transitions is that the rotational quantum number $N$ in the ground state can only differ from $N$ in the excited state 


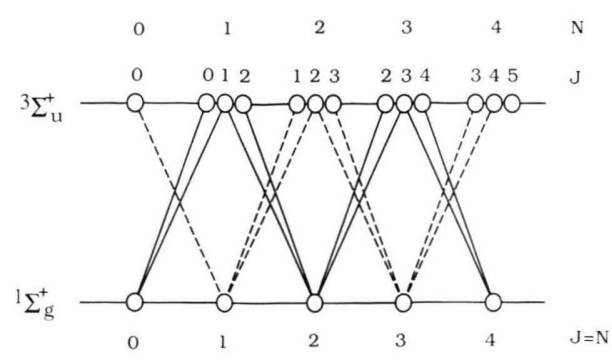

Fig. 3. Schematic representation of the transitions of the ${ }^{3} \Sigma_{\mathrm{u}}^{+}-\mathrm{X}^{1} \Sigma_{\mathrm{g}}^{+}$band in ${ }^{199} \mathrm{Hg}_{2}$ dimer. The solid (broken) lines refer to transitions between nuclear singlet (triplet) states. Due to the electronic triplet character of the upper state the rotational quantum number $N$ is not identical with the total angular momentum quantum number $J$. The order of the levels for fixed $N$ is arbitrary (after Herzberg [7] p. 276).

by \pm 1 . Thus, since transitions between anti-symmetric and symmetric rotational levels are strongly forbidden, it is the change in the symmetry behavior between the rotational levels in the two electronic states $\left({ }^{1} \Sigma_{\mathrm{g}}^{+}\right.$and $\left.{ }^{3} \Sigma_{\mathrm{u}}^{+}\right)$that allows transitions between these electronic states of the ${ }^{199} \mathrm{Hg}_{2}$ dimer (c.f. Figure 3).

In conclusion, only nuclear singlet states are dissociated by using transitions starting with even $N$. Specifically, due to the angular momentum selection rules, $\Delta N= \pm 1$, for the excitation $(266 \mathrm{~nm})$ and stimulated emission $(355 \mathrm{~nm})$ transitions the final dissociating level of the $X^{1} \Sigma_{\mathrm{g}}^{+}$ground state must also have even $N$ and hence zero total nuclear spin. As it turns out, the $P(10)$ transition is particularly favorable, both because the rotational state population has a peak at $N=10$ and because it is well resolved [6].

It should be mentioned that for the electronic excited state the electronic state designation ${ }^{3} \Sigma_{u}^{+}$has been used, which, in principle, is only valid for an angular momentum coupling scheme of Hund's case (a) or (b). Since, however, $\mathrm{Hg}_{2}$ is a van der Waals molecule, Hund's coupling case (c) with the notation $1_{\mathrm{u}}$ would be more appropriate. However, the work by Hay et al. [8] and a review article by Morse [9] indicate that the $\mathrm{D}\left(1_{\mathrm{u}}\right)$ mainly has a ${ }^{3} \Sigma_{\mathrm{u}}^{+}$state character. Even if a mixing of the ${ }^{3} \Sigma_{\mathrm{u}}^{+}$with the other possible admixture to a $1_{\mathrm{u}}$ state (the ${ }^{1} \Pi_{\mathrm{u}}^{+}$state) is considered, the selection of a spin singlet state is still possible. Specifically, all rotational levels in a ${ }^{1} \Pi_{\mathrm{u}}^{+}$state show $\Lambda$-doubling into a symmetric and anti-symmetric component. Since the selection rules only allow transitions between states of the same total symmetry, it is still possible to dissociate only molecules in a nuclear spin singlet state [7].

\section{Test of the $\mathrm{BCH}-$ Inequality}

The two resulting mercury atoms are in ${ }^{1} \mathrm{~S}_{0}$ ground states. Due to the nuclear spin $I=1 / 2$ of ${ }^{199} \mathrm{Hg}$, each ground state atom has a total angular momentum $F=1 / 2$ with components $\pm 1 / 2$. Consequently, we will be observing correlations between components of the spin of two spatially separated spin- $1 / 2$ particles. The nuclear spin component analysis is accomplished via electric dipole transitions [6]. The test of the $\mathrm{BCH}$ inequality requires the measurement of coincidence rates $R_{++}\left(\theta_{1}, \theta_{2}\right)$ for the simultaneous detection of an atom at detector 1 with $M_{F}=+1 / 2$ in the direction $\theta_{1}$ and of an atom at detector 2 with $M_{F}=+1 / 2$ in the direction $\theta_{2}$ as well as of the singles rates $R_{i+}\left(\theta_{i}\right)$, which are defined as the rate of detection of atoms with $M_{F}=+1 / 2$ in the direction $\theta_{i}$ at detector $i$, where $i=1$ or 2 . The $\mathrm{BCH}$ inequality is formulated in terms of the ratio of coincidence rates to singles rates for four different angles with respect to which the spin state has to be determined $[4,5]$. In the present context, the $\mathrm{BCH}$ inequality can be expressed as

$S\left(a, b, a^{\prime}, b^{\prime}\right)$

$=\frac{R_{++}(a, b)-R_{++}\left(a, b^{\prime}\right)+R_{++}\left(a^{\prime}, b\right)+R_{++}\left(a^{\prime}, b^{\prime}\right)}{R_{1+}\left(a^{\prime}\right)+R_{2+}(b)} \leq 1$,

where $a, a^{\prime}$ are two values of angles for the first detector and $b, b^{\prime}$ are two values of angles for the second detector.

Real world analyzers, however, have an efficiency $\varepsilon_{M}$ less than unity for one spin state and a leakage $\varepsilon_{m}$ greater than zero for the other spin state. The influence of these imperfections on the measurement of coincidence rates will be discussed.

Instead of only measuring one well defined coincidence, these imperfections lead to a mixing of the four possible outcomes of the coincidence measurement. The measured rate $R_{++}\left(\theta_{1}, \theta_{2}\right)$ is a combination of the ideal rates $R_{++}^{\prime}\left(\theta_{1}, \theta_{2}\right), R_{--}^{\prime}\left(\theta_{1}, \theta_{2}\right), R_{+-}^{\prime}\left(\theta_{1}, \theta_{2}\right)$, and $R_{-+}^{\prime}\left(\theta_{1}, \theta_{2}\right)$ :

$$
\begin{aligned}
R_{++}\left(\theta_{1}, \theta_{2}\right)= & \varepsilon_{M}^{2} R_{++}^{\prime}\left(\theta_{1}, \theta_{2}\right)+\varepsilon_{m}^{2} R_{--}^{\prime}\left(\theta_{1}, \theta_{2}\right) \\
& +\varepsilon_{M} \varepsilon_{m} R_{+-}^{\prime}\left(\theta_{1}, \theta_{2}\right)+\varepsilon_{M} \varepsilon_{m} R_{-+}^{\prime}\left(\theta_{1}, \theta_{2}\right),
\end{aligned}
$$

where the subscripts + and - indicate $m_{F}=1 / 2$ (spin up) and $m_{F}=-1 / 2$ (spin down), respectively. For sim- 
plicity the same imperfections $\varepsilon_{M}, \varepsilon_{m}$ were assumed for both detectors.

As we showed previously [6], the quantum mechanical predictions for the four possible outcomes of coincidence measurements with respect to two angles $\theta_{1}$ and $\theta_{2}$ are

$$
\begin{aligned}
R_{++}^{\prime}\left(\theta_{1}, \theta_{2}\right) & =R_{--}^{\prime}\left(\theta_{1}, \theta_{2}\right) \\
& =\eta^{2} f g N \frac{1}{4}\left[1-\cos \left(\theta_{1}-\theta_{2}\right)\right], \\
R_{+-}^{\prime}\left(\theta_{1}, \theta_{2}\right) & =R_{-+}^{\prime}\left(\theta_{1}, \theta_{2}\right) \\
& =\eta^{2} \text { fg } N \frac{1}{4}\left[1+\cos \left(\theta_{1}-\theta_{2}\right)\right],
\end{aligned}
$$

where $\eta$ is the detector efficiency of $\mathrm{Hg}$ atoms arriving at the corresponding detectors; the two detectors are symmetric and have identical geometries with an acceptance solid angle $f ; g$ is the conditional probability that if one of the atoms from a dissociated dimer enters the aperture of one detector, then the other atom from that dimer enters the aperture of the other detector.

With the help of (4) and (5), (3) can be rewritten in the form

$R_{++}\left(\theta_{1}, \theta_{2}\right)=\eta^{2} f g N \frac{1}{4}\left[\varepsilon_{+}^{2}-\varepsilon_{-}^{2} \cos \left(\theta_{1}-\theta_{2}\right)\right]$,

where $\varepsilon_{+}$and $\varepsilon_{-}$are defined as $\varepsilon_{+}=\varepsilon_{M}+\varepsilon_{m}$ and $\varepsilon_{-}=\varepsilon_{M}-\varepsilon_{m}$, respectively.

Similarly, by using the quantum mechanical predictions for a homogenous distribution of the spin states [6],

$R_{1+}^{\prime}\left(\theta_{1}\right)=R_{2+}^{\prime}\left(\theta_{2}\right)=R_{1-}^{\prime}\left(\theta_{1}\right)=R_{2-}^{\prime}\left(\theta_{1}\right)=\frac{\eta f N}{2}$,

one finds for the singles count rate

$R_{1+}\left(\theta_{1}\right)=R_{2+}\left(\theta_{2}\right)=\frac{\eta f N}{2} \varepsilon_{+}$.

These QM predictions, (6) and (8), can be used to calculate the maximum violation of the $\mathrm{BCH}$ inequality by quantum mechanics. The maximum is reached for $a, b, a^{\prime}, b^{\prime}$ equal to $135^{\circ}, 0^{\circ}, 225^{\circ}$, and $90^{\circ}$, respectively,

$S_{\mathrm{QM}}\left(135^{\circ}, 0^{\circ}, 225^{\circ}, 90^{\circ}\right)=\frac{1}{2} \eta g\left(\varepsilon_{+}+\sqrt{2} \frac{\varepsilon_{-}^{2}}{\varepsilon_{+}}\right)$.

In the case of perfect analyzers, i.e. $\varepsilon_{+}=\varepsilon_{-}=1$, this result reduces to $S_{\mathrm{QM}}\left(135^{\circ}, 0^{\circ}, 225^{\circ}, 90^{\circ}\right)$ $=\frac{1}{2}(1+\sqrt{2}) \eta g \approx 1.207 \eta g$, as derived previously [6]. Clearly, in order to get the highest possible violation of the $\mathrm{BCH}$ inequality by quantum mechanics, the right hand side of (9) must be as large as possible.
The conditional probability $g$ is determined by the size of the dissociation volume, the angular distribution of the dissociating dimer fragments, the size and position of the detectors as well as the spread in the velocities of the dissociating fragments. Monte-Carlo simulations show that for the parameters anticipated in our experiment a value of $g=0.976$ is expected [6].

The second requirement for the test of the $\mathrm{BCH}$ inequality is the highest possible detection efficiency $\eta$. The atomic level structure of $\mathrm{Hg}$ permits a two-photon resonant photo-ionization scheme [6]. $\mathrm{Hg}$ atom detection can thus be achieved via both the resulting ion and the photoelectron in an OR-detection scheme, which means that the $\mathrm{Hg}$ atom is detected if either the ion or the associated photoelectron is detected. Employing the OR-detection scheme, detector efficiencies larger than $95 \%$ are expected. Actually, overall $\mathrm{Hg}$ atom detection efficiencies $>99 \%$ should be achievable.

Finally a very high discrimination of the analyzers between the two different nuclear spin states must be achieved. In fact by using the appropriate laser polarizations in the two-photon excitation/ionization scheme a near perfect discrimination is possible [6]. Simulations show that values of $99.5 \%$ for the transmission $\varepsilon_{M}$ can be reached. In addition the rejection $\varepsilon_{m}$ is extremely high $\left(\varepsilon_{m}<0.075 \%\right)$.

The quantum mechanical prediction $S_{\mathrm{QM}}$ of (9) can now be evaluated by using the values $g=0.976$, $\eta=0.95, \varepsilon_{+}=0.996$, and $\varepsilon_{-}=0.994$ :

$$
S_{\mathrm{QM}}\left(135^{\circ}, 0^{\circ}, 225^{\circ}, 90^{\circ}\right)=1.112 \text {. }
$$

Thus the $\mathrm{BCH}$ inequality is clearly violated. The parameters of our experiment provide a definitive test of the $\mathrm{BCH}$ inequality, that closes the detection loophole even considering non-perfect analyzers. The second remaining loophole, the enforcement of the locality condition, can also be closed employing electro-optic modulators [6].

\section{Acknowledgement}

This research was supported by the Robert A. Welch Foundation under Grant No. A-1218, by the Texas Advanced Research Program under Grant No. 010366-017, and by the National Science Foundation under Grant PHY-9221038. The authors thank John Clauser, Shifang Li, and Marlan Scully for many helpful discussions and encouragement and Jason Pan for his active participation. 
[1] J. S. Bell, Physics 1, 195 (1964).

[2] A. Einstein, B. Podolsky, and N. Rosen, Phys. Rev. 47, 777 (1935).

[3] J. F. Clauser, M. A. Horne, A. Shimony, and R. A. Holt, Phys. Rev. Lett. 23, 880 (1969).

[4] J. F. Clauser and A. Shimony, Rep. Prog. Phys. 41, 1881 (1978).

[5] J. F. Clauser and M. A. Horne, Phys. Rev. D 10, 526 (1974).
[6] E. S. Fry, Th. Walther, and S. Li, Phys. Rev. A 52, 4381 (1995).

[7] G. Herzberg, Molecular Spectra and Molecular Structure, (D. Van Nostrand Company, Inc., Princeton, New Jersey, 1950)

[8] P. J. Hay and J. T. H. Dunning, J. Chem. Phys. 65, 2679 (1976).

[9] M. D. Morse, Chem. Rev. 86, 1049 (1986). 